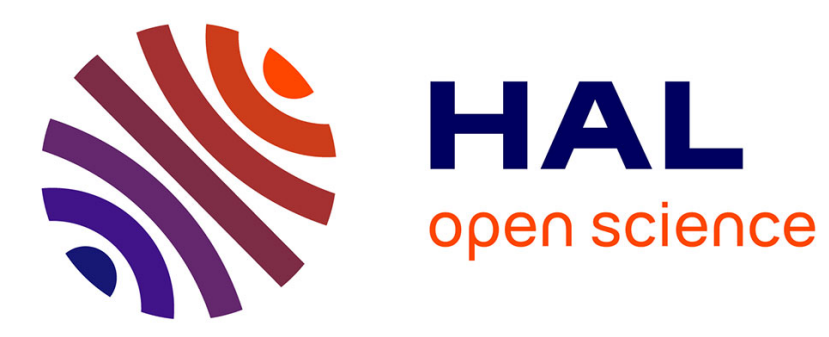

\title{
Balthus et la scène pubertaire féminine
}

Nicolas Rabain, Isée Bernateau

\section{To cite this version:}

Nicolas Rabain, Isée Bernateau. Balthus et la scène pubertaire féminine. Adolescence, 2015, Passion, 33 (1), pp.207 - 218. 10.3917/ado.091.0207 . hal-01411223

\section{HAL Id: hal-01411223 \\ https://hal.science/hal-01411223}

Submitted on 9 Dec 2016

HAL is a multi-disciplinary open access archive for the deposit and dissemination of scientific research documents, whether they are published or not. The documents may come from teaching and research institutions in France or abroad, or from public or private research centers.
L'archive ouverte pluridisciplinaire HAL, est destinée au dépôt et à la diffusion de documents scientifiques de niveau recherche, publiés ou non, émanant des établissements d'enseignement et de recherche français ou étrangers, des laboratoires publics ou privés. 


\section{Balthus et la scène pubertaire féminine}

Nicolas Rabain, Isée Bernateau

Longs après-midi d'enfance, Pas vraiment vivant encore,

Toujours en maturation,

En proie à des peines grandissantes, Exposé sans défense à de longues attentes.

[...] Après-midi solitaires, A se contempler d'un miroir à l'autre. ${ }^{2}$

Le temps de l'enfance ${ }^{3}$ Rainer Maria Rilke

Dans le catalogue de l'exposition Présumés innocents, Henry-Claude Cousseau -exdirecteur des Musées de Bordeaux - rappelle la part centrale que le vingtième siècle a donnée à l'enfance en tant que "source d'inspiration sans précédent dans l'histoire de l'art occidental ${ }^{4}$. Si les époques ultérieures regorgent de sujets liés à l'enfant, seule la nôtre aurait su lui accorder une place «aussi intimement liée à son existence, à ses préoccupations, à sa propre quête [...] Ce ne sont plus tant les traces balbutiantes, émouvantes, de son ingénuité qu'on perçoit, que les signes de ce que l'enfant paraît receler au fond de lui-même, de ce que ses jeux et ses comportements livrent de rêves, de fantasmes comme de part d'ombre. » ${ }^{5}$ Remanier cetteassertion, en remplaçant le terme d'«enfant» par celui d' ' adolescent», nous permet d'entrevoir quelques éléments de réponse à la principalequestionposée parPhilippe Gutton dans son dernier ouvrage : que viendraient nous révéler les toiles de Balthasar Klossowski (1908-2001) - alias Balthus -au sujet de l'adolescence ?DansBalthus et les jeunes filles - ou le dévoilement du féminin, Philippe Guttonnous invite à revisiter certains concepts psychanalytiques qu'il a lui-même forgés après plus de trente ans de travail auprès de jeunes patients. L'abord des toiles du peintreest pour lui l'occasion de mettre à l'épreuve sa conception de l'adolescence en tant que période de créativité subjectale sous l'emprise de la nécessité pubertaire.Autant chez le peintre que chez le psychanalyste, la métamorphose pubertaire féminine paraît constituer sinon un sujet de fascination, du reste un point de convergence qui permettrait de confronter les représentations picturales de l'un aux avancées théoriques de l'autre.

\footnotetext{
${ }^{1}$ A partir du livre de Philippe Gutton (2013).Balthus et les jeunes filles - ou le dévoilement du féminin. Paris : Editions EDK, 203 pp.

2, Lange Nachmittage der Kindheit / Immer noch nicht Leben / Immer noch Wachstum / Das in den Knien zieht / Wehrlose Wartezeit [...] / Nachmittage, da es allein blieb / Von einem Spiegel zum andern starrend - Dauer der Kindheit "(Traduit par nous).

${ }^{3}$ Rilke R. M. (1924). Dauer der Kindheit.In : Rainer Maria Rilke Werke - Band 2 : Gedichte 1910 bis

1926. (Briefwechsel mit Erika Mitterer in Die Gedichte 1922 bis 1926). Frankfurt am Main : Insel ; 1996 , p. 337. ${ }^{4}$ Présumés innocents - L'art contemporain et l'enfance, exposition du 8 juin au $1^{\mathrm{er}}$ octobre 2000 , capcMuséed'art contemporain de Bordeaux, p.7.

${ }^{5}$ CousseauH.-C. (2000). Préface. In :Présumés innocents - L'art contemporain et l'enfance, Bordeaux : Réunion des Musées Nationaux, pp. 7-8.
} 
Comme l'ont souligné Deleuze et Guattari, «Ce n'est pas la jeune fille qui devient femme, c'est le devenir-femme qui fait la jeune fille universelle. ${ }^{6}$ Serait-ce ainsi par l'adolescence que Balthus rendrait visible l'invisible de la femme ? Que voit Balthus de la femme en l'adolescente ? Comment le transmet-il à travers ses œuvres ?Dans cette réflexion sur la création de la femme, il va de soi que l'adolescente n'est pas envisagée comme un canon jeune de la beauté classique. Au contraire, elle est abordée sous l'angle d'un être incomplet sur le point d'atteindre un stade de complétude. Ce que Balthus exprime ainsi : «Je vois l'adolescente comme un symbole. Je ne pourrais jamais peindre une femme. La beauté de l'adolescente est plus intéressante. L'adolescente incarne l'avenir, l'être avant qu'il ne se transforme en beauté parfaite. Une femme a déjà trouvé sa place dans le monde, une adolescente, non. Le corps d'une femme est déjà complet. Le mystère a disparu. ${ }^{7}$

\section{La psychanalyse face aux jeunes filles de Balthus}

Rappelons qu'en 1991, Philippe Gutton expose le concept de «pubertaire » dans son célèbre ouvrage éponyme. Il en donne la définition suivante : « Le mot puberté est au corps ce que le pubertaire est à la psyché ${ }^{8}$.Ce concept clef siège au cœur de l'édifice théorique du psychanalyste, notamment dans la subdivision en deux temps qu'il fait du processus adolescent : d'après lui, on peut d'abord observer le temps du saisissementlié à la mutation narcissico-pulsionnelle, caractéristique du pubertaire; dans un second temps, avance-t-il, untravail actif de créationvise à élaborer ce que le surgissement du pubertaire est venu ébranler. Cette élaboration s'appuieraitmajoritairement sur des éléments issus del'infantile. Ainsi, la métamorphose pubertaire est décrite ici comme un point de départ d'une nouvelle subjectalisation où les organisations infantiles entrent en mutation et se remanient.

Ce travail de création est à nouveau évoqué par Philippe Gutton dès le début de son livresur Balthus. Il y relève certaines similitudes entre les artistes et les adolescents face auprocessus de création. Tout comme l'adolescent, l'artiste souhaiterait en effet représenter par une rhétorique ce qu'il ressent passionnément. L'auteur attire l'attention sur deux notions impliquées dans le processus de création: d'une part l'emprise, en lien avec la volonté figurative du peintre, exigeant l'élaboration d'une production structurée ; et d'autre part, la sublimation, impliquant un va-et-vient entre le sensoriel et le mécanisme de symbolisation. Aussi insiste-t-il finalement sur l'idée que la sublimation de l'artiste est soumise à l'emprise de l'artisan. Il est donc pertinent d'appréhender création artistique et génie adolescent comme deux entités qui se recoupent. En l'occurrence, le saisissement de Balthus proviendrait, d'après Philippe Gutton, de la séduction esthétique qu'il ressentirait face aux adolescentes. Dans cette perspective, il resterait proche de l'expérience originaire que l'adolescence symbolise. Ainsi, grâce à ses modèles, Balthus créerait des représentations singulières qui auraient pour particularité de mettre en relief ce qu'il y a derrière l'apparence.Cette activité est propre au travail des peintres qui, comme l'a souligné Barthes, auraient pour dessein de «montrer » sans pour autant « démontrer ». ${ }^{9}$

Si l'artiste invente en quelque sorte ce qui existe déjà, son œuvre mettrait parallèlement en scène l'advenue du féminin pubertaire dans un contexte de féminité infantile phallique. C'est la thèse que Philippe Gutton va soutenir. En effet, si la petite fille fait face à une problématique de castration féminine - l'avoir -, le féminin pubertaire tient quant à lui à

\footnotetext{
${ }^{6}$ Deleuze G. et Guattari F. (1980). Mille Plateaux. Paris : Editions de Minuit, p.339.

${ }^{7}$ Entretiens avec Richard Gèze. In : Collectif (2008). Balthus - Portraits privés. Lausanne : Editions Noir sur Blanc, p. 68.

${ }^{8}$ Gutton Ph. (1991).Le pubertaire. Paris : Editions PUF - Le fil rouge ; p. 7.

${ }^{9}$ Barthes R. (1970).L'empire des signes. Paris : Editions Flammarion.
} 
une sensorialité génitale nouvelle : l'être avec.Ainsi, l'accent est mis sur le passage de la féminité infantile à la féminité pubertaire. La petite fille est sous l'emprise de la logique narcissique phallique et se retrouve, d'après Philippe Gutton, «[...] sollicitée par son corps en tant que complémentaire de l'autre sexe [...] » avec l'émergence du phénomène pubertaire. ${ }^{10}$ Par ailleurs, l'auteur nous rappelle que la rencontre entre féminin pubertaire et féminité infantile phallique se trouve mise en tension dans le travail de subjectalisation dans la scène adolescente. Dans certains tableaux,les postures des jeunes filles révèleraient le dualisme entre infantile et génitalité, notamment dans l'alternance entre les adolescentes allongéeset celles qui ont été peintes debout.

La scène pubertaire est ainsi entrevue comme la reprise active brute de la scène primitive à cette différence près que l'enfant, jadis passivé, devient ici actif. Notons ici que ce renversement de la passivation infantile en activation est régulièrement représenté par Balthus à proximité d'objets contondants pouvant faire écho eux-mêmes à la parentalité phallique, tantôt protectrice tantôt menaçante. Ainsi, Philippe Gutton soutient l'idée que cette scène pubertaire est agiedans le champ de la psychose et de la perversion. Quant à son élaboration, elle conduirait au contraire à des scènes adoucies et organisées que sont les scènes adolescentes proprement dites. Il serait alors question d'élaborations œdipiennes plus névrotiques supposant la mise à distance progressive d'imagos parentales séductrices, accompagnée d'une pacification des liens avec le parent du même sexe. Toutefois, avant d'aboutir à ce remaniement des imagos parentales qui signerait la fin de l'adolescence, nous sommes avec Balthus face à des représentations d'une «encore-enfant» dans la problématique de la castration sous le regard phallique.

Parallèlement,Philippe Gutton développe un aspect théorique des plus éclairants pour tout psychothérapeute d'adolescents. D'après lui, c'est essentiellement le regard de l'autre et notamment celui de l'adulte - quidétournerait de l'innocence. Il rappelle à cet égard que «La dimension dramatique de l'adolescence est toute entière associée à l'emprise du regard phallique. ${ }^{11} \mathrm{Or}$, dans ce moment critique où le pubertaire en excès ne peut être entièrement tempéré par l'infantile sexuel, le regard de l'adulte va venir faire effraction - au sens où l'entend Ferenczi dans La confusion de langue entre l'adulte et l'enfant. ${ }^{12} \mathrm{En}$ somme, ce que l'adolescente est et devient résiderait dans les yeux qui la regardent.Cette dualité entre tendresse et génitalité se retrouve dans les tableaux de Balthus à travers deux catégories de références contraires : l'ange et la bête ; l'infantile et le pubertaire ; ou encore, «Le dard et la fleur $»^{13}$ comme le commentait René Char.Si la violence à l'adolescence émane en grande partie d'un choc entre ces deux courants contraires - organisation infantile installée et génitalité advenante - elle n'en reste pas moins génératrice de créativité.

Dans une troisième et dernière partie, le psychanalyste met la lumière sur la première scène qui se déroule entre le peintre, son modèle et l'œuvre. De la rencontre entre le modèle et l'artiste naît l'inspiration. Il est probable qu'un modèle interne soit déjà présent sous une autre forme dans la réalité psychique de l'artiste sans qu'il soit encore connu pour autant. Le modèle viendrait révéler le «déjà là » chez l'artiste et par là même, l'accompagner dans la création de la scène adolescente. En somme, pas plus de mères sans bébés que d'artistes figuratifs sans modèles. Dans ce dispositif où la séduction revêt une place primordiale, Balthus est alors en mesure de dévoiler en tant qu'adulte ce qu'il connaît et ce qu'il reconnaît

\footnotetext{
${ }^{10}$ Gutton Ph. (2013), op. cit., p. 76.

${ }^{11}$ Ibid., p. 100.

${ }^{12}$ Ferenczi S. (1932). Confusion de langue entre les adultes et l'enfant. In :Euvres complètes - Tome IV, (19271933).Paris :Payot ; 1982, pp. 125-135.

${ }^{13}$ Char R. (1946).Balthus ou le dard dans la fleur. In : Revue des Cahiers d'art1945-1946 - numéro unique - 20 et 21èmes années, p. 199.
} 
$\mathrm{du}$ désir. D'après $\mathrm{Ph}$. Gutton, cette «pédagogie amoureuse » serait commune à la fois aucélèbre peintre des jeunes filles et aux psychanalystes d'adolescents.

Ainsi, la scène pubertaire féminine- telle que l'a développée Philippe Gutton au fil de son œuvre - a pu être revisitée après avoir été confrontée aux toiles de Balthus où figurent des jeunes filles. Toutefois, si les tableaux peuvent être appréhendés comme des œuvres proposant des mises en scène à décrypter, nous pourrions également les considérer comme des supports visuels invitant chacun à créer de nouvelles chaînes associatives. Cette invitation concerne tout autant le peintre et le visiteur de musées que les jeunes filles elles-mêmes.

Or, dans son livre, le psychanalyste semble essentiellement identifié à l'artiste, ce qui le conduit à ne pas donner la priorité au point de vue des jeunes filles. Dans quelle mesure l'auteur ne néglige-t-il pas une partie de l'œuvre de Balthus, en restant essentiellement centré sur ses propres concepts psychanalytiques ? Après avoir commenté le regard du peintre ou celui du psychanalyste sur les jeunes filles, il faut maintenant se pencher sur le point de vue des adolescentes en nous demandant à quoi ellesrêvent.

\section{Les jeunes filles face à Balthus et aux psychanalystes}

Un psychanalyste écrit sur l'œuvre d'un artiste, et plus particulièrement sur les jeunes filles que ce dernier a peintes. En effet, dans l'ouvrage de Philippe Gutton, l'éclairageest essentiellement mis sur la manière dont Balthus représente les adolescentes qu'il a choisies pour modèles. L'angle de vue est en somme celui de deux hommes observant des jeunes filles. Qu'en est-il cependant du point de vue des modèles de Balthus ?A quoi rêvent les adolescentes qui ont posé dans son atelier ? Comment ont-elles évolué sachant que certaines d'entre elles ont travaillé aux côtés du peintre des années durant ? (Anna ou encore Thérèse, pour ne citer qu'elles).CarPhilippe Gutton ne questionne pas l'évolution des représentations picturales de Balthus au fil du temps. Il est davantage question de considérer ce que l'artiste nous apprend sur la scène pubertaire féminine. Le contexte historique ou encore socio-culturel dans lequel ces jeunes femmes vivent est quant à lui peu pris en ligne de compte.

Dans l'analyse que fait Philippe Gutton des toiles de Balthus, une dimension intemporelle l'emporte. Autrement dit, l'intemporalité dans laquelle seraient représentées les jeunes filles de Balthus conduit le psychanalyste d'adolescents à formuler des hypothèses plus générales. Ce parti pris, aussi pertinent soit-il, laisse dans l'ombre un certain nombre de questions que l'artiste avait peut-être en tête. Pour lui, ses modèles correspondaient-ils à des stéréotypes ou à un archétypes de la jeune fille ? En d'autres termes, Balthus prenait-il appui sur ses modèlespour faire prévaloir le singulier ou l'universel ?Ainsi, d'où vient l'apparente lassituded'un grand nombre de jeunes filles peintes par Balthus.L'artiste cherche-t-il àreprésenter une allégorie de la morosité à l'adolescence ouà faire le portrait d'une jeune fille particulière et de ses singularités?

$\mathrm{Si}$ certaines adolescentes paraissent prostrées, voire privées de toute activité fantasmatique, d'autres au contraire sont en pleines rêveries diurnes. Cette opposition entre vide psychique et ressources associatives ferait également écho à la personnalité des modèles et aux liens que le peintre aurait tissés au fil du temps avec chacune d'elles.Dans un article qu'elle consacre à Thérèse Blanchard ${ }^{14}$ - modèle de Balthus de 1936 à 1939 - Sabine Rewald, critique d'art, centre son propos sur les « adolescentes isolées dans des chambres fermées au monde extérieur. $»^{15}$ D'après elle, Balthus représente souvent une jeune fille qui concentre en

\footnotetext{
${ }^{14}$ Rewald S. (1998).Balthus'sThérèses.In :Metropolitan Museum Journal, vol. 33. The University of Chicago Press, pp. 305-314.

${ }^{15}$ Ibid., p.309: " [...] adolescents isolated in rooms closed to the outside world"(Traduit par nous)
} 
elle à la fois «lassitude et exubérance, innocence et fantasmes sexuels, rêve et réalité. »16 Fréquemment, Balthus joue sur un dualisme qui revêt plusieurs formes. S. Rewald évoque « une dichotomie entre le désir de Balthus face aux adolescentes et parallèlement, son empathie vis-à-vis d'elles ». Cette dichotomie trouverait une analogie formelle, avance-t-elle, «dans leurs postures excitantes qui par ailleurs suivent scrupuleusement un ordre pictural d'une grande rigueur architecturale. » ${ }^{17}$

Cette double posture du peintre face aux jeunes filles paraît faire écho à un grand nombre de représentations de couples d'opposés, à commencer par le sadisme et le masochisme. Il est au cœur de la célèbre toile du maître, copieusement controversée : $L a$ leçon de guitare (1934). Un autre pôle antagoniste et complémentaire revient tel un leitmotiv chez Balthus : l'opposition entre activité et passivité. Elle est fréquemment illustrée par des garçons dans des rôles passifs alors que «les filles et les chats dominent. » ${ }^{18}$ Ainsi, la question du sadomasochisme et l'alternance entre la passivité et l'activité sont autant d'éléments représentés par Balthus qui percevrait à la fois l'émergence de la sexualité génitale chez les adolescentes tout en projetant en partie la sienne.C'est l'une des principales raisons pour lesquelles l'œuvre du peintre a souvent été qualifiée de sulfureuse par certains, voire de scandaleuses par d'autres. Récemment, le Musée Folkwang d'Essen a suspendu une exposition de deux mille Polaroid pris par Balthus. Au cœur du scandale, l'une des ses modèles favorites: Anna, photographiée entre ses huit et ses seize ans "parfois un peu déshabillée » en guise d'études préparatoires à de futures toiles. A cette occasion, l'hebdomadaire allemand dieZeit a publié un article calomnieux qualifiant ces photos de « témoignages de l'avidité d'un pédophile. » 19

Cette vague de contestation rappelle il y a quelques années l'accueil de la série de photos de Garry Gross ${ }^{20}$ intitulée The Woman in the Child ou encore, un scandale de même nature faisant suite à l'exposition des photos de Larry Clark. ${ }^{21}$ L'une des spécificités de leurs œuvres consistait également en l'équation : en la fillette, la femme ; en l'adolescent, l'homme. Si d'aucuns ont cru bon de jeter l'opprobre sur ses œuvres - et en l'occurrence, sur celle de Balthus - nombreux sont ceux qui se sont intéressés et parfois inspirés d'elle: artistes plasticiens, écrivains, poètes, cinéastes ou encore critiques d'art. Car l'œuvre de Balthus naît justement à une époque où les représentations de jeunes filles connaissent un tournant majeur. Rappelons ici que dans les années vingt, la popularisation des revues et de la photographie permet une multiplication conséquente des images de jeunes filles. Il faut cependant attendre le début des années cinquante pour que le magazine Elle joue sur la notion de «règne de l'adolescente » qui devient rapidement un modèle pour les médias, notamment à travers les publicités. Plus tard, dans les années soixante, une véritable explosion de la «culture jeune » s'accompagne de la crise de la jeune fille, notamment en ce qui concerne l'apprentissage de la féminité. Jusqu'alors, les garçons étaient considérés à la fois comme les décodeurs de l'histoire, et le futur de la reconstruction; quant aux femmes, elles assuraient la stabilité culturelle. Soulignons encore qu'auparavant, les jeunes filles n'avaient pas de place définie. ${ }^{22}$ Balthus aurait-il permis à travers ses œuvres une remise en question de la place

\footnotetext{
${ }^{16}$ Ibid., p. 305 : "[...] that stage of life that veers between lassitude and exuberance, innocence and sexual fantasies, reality and dream." (Traduit par nous)

${ }^{17}$ Ibid., p.307: “" The dichotomy between the painter's desire for and empathy with the adolescent girl finds a formal analogy in her titillating posture, which is then severely disciplined by a pictorial order of architectural rigor. "(Traduit par nous)

${ }^{18}$ Ibid.,p.311: “[ ...] girls and cats dominate” (Traduit par nous)

${ }^{19}$ Balthus censuré en Allemagne. In : Le Monde daté du samedi 22 février 2014.

${ }^{20}$ Gross G. (1975). The Woman in the Child. New York: American Fine Arts.

${ }^{21}$ Clark L. (1992-1995).Untitled. Série de photographies, 32 tirages couleurs. New York : Galerie Luhring Augustine.

${ }^{22}$ Lebovici E. (2013).Contribution au séminaire Arts et féminismes. Antibes : Fondations Hartung-Bergman.
} 
accordée aux jeunes filles de son époque? Il semblerait en réalité que ses principales préoccupations se jouaient ailleurs.

\section{Conclusion}

Afin de mettre à l'épreuve certains de ses propres outils conceptuels, Philippe Gutton, dans Balthus et les jeunes filles, s'est confronté aux toiles d'un artiste pourtant connu pour ses réticences personnelles à l'égard de la psychanalyse. Ainsi, Gutton a travaillé au repérage d'un savoir inconscient universel siégeant dans les toiles d' « un homme dont on ne sait rien $\gg .{ }^{23}$ Pour autant, cet homme réfractaire à toute considération psychanalytique est aussi celui qui figure non pas ce qui est là, mais ce qui devient. Si le féminin pubertaire se révèle par des transformations visibles, Balthus questionne quant à lui l'invisible féminin en peignant des scènes ambiguës dans lesquelles le modèle cacherait la femme et, paradoxalement, la serait à la fois toute entière. Le corps de la femme prend ici les allures d'une métaphore à tout penser. L'histoire de l'art nous rappelle à cet égard que la jeune fille vierge a souvent été perçue comme une métaphore de la toile «vierge » que le peintre viendrait recouvrir.

Le pouvoir évocateur des toiles conjugué aux théories psychanalytiques du processus adolescent permet d'appréhender un certain nombre de questions cliniques. Parmi ces questionnements, la subjectivation adolescente est au cœur du propos. L'adolescence y est entrevue comme une phase éphémère « a-phallique ». Philippe Gutton s'avance même jusqu'à formuler que le fait pubertaire permet d'échapper à une topologie exclusivement phallique en construisant une génitalité «personnelle». Ainsi, la subjectivation à l'adolescence ne viendrait pas cliver les deux sexualités humaines (tendre et génitale) comme certains auteurs ont pu le soutenir. Au contraire, elle cliverait ces deux sexualités de la phallicité infantile.

Dans un article intitulé «Régression digressions, récréation ${ }^{24}$, Elisabeth Lebovici pose la question suivante : «Faire l'enfant, mimer l'adolescence, donner dans la gaminerie, sont-ce des traits spécifiques aux œuvres ou à leurs auteurs ? [...] La question est : qui fait l'enfant ? ${ }^{25}$ Dans cette perspective, on peut se demander ce qui appartient à Balthus, ou plus encore, ce qu'il laisse échapper de sa propre existence lorsqu'il représente des jeunes filles. Philippe Gutton tente de répondre à cette énigme en avançant que la problématique sadomasochiste de la phallicité, centrale chez la jeune fille, serait convoquée chez Balthus et renforcerait en lui une angoisse de castration. Ne serait-ce justement pas son activité artistique qui lui aurait permis de colmater en partie cette angoisse ?

Nicolas Rabain, Isée Bernateau

CRPMS

UFR Etudes psychanalytiques Université Paris Diderot Bâtiment Olympe de Gouges

8 rue Albert Einstein 75013 Paris

\footnotetext{
${ }^{23}$ Texte de présentation de Balthus par lui-même à l'occasion d'une rétrospective à la Tate Gallery.

${ }^{24}$ Lebovici E. (2000). Régression, digressions, récréation. In : Présumés innocents - L'art contemporain et l'enfance. Bordeaux : Réunion des Musées Nationaux, pp. 134-139.

${ }^{25}$ Ibid., p. 136.
} 


\section{Bibliographie}

- Barthes R. (1970).L'empire des signes. Paris :Editions Flammarion.

- Char R. (1946).Balthus ou le dard dans la fleur. In : Revue des Cahiers d'art19451946 - numéro unique - 20 et 21èmes années, p. 199.

- Collectif (2008). Balthus - Portraits privés. Lausanne : Editions Noir sur Blanc.

- Collectif (2000). Présumés innocents - L'art contemporain et l'enfance. Bordeaux :capcMusée d'art contemporain de Bordeaux, Editions Seuil, 176 pp.

- Cousseau H.-C.,Préface, pp. 7-8.

- Lebovici E., Régression, digressions, récréation, pp. 135-139.

- $\quad$ Deleuze G. et Guattari F. (1980).Mille Plateaux. Paris :Editions de Minuit, 339 pp.

- Ferenczi S. (1932). Confusion de langue entre les adultes et l'enfant. In :Euvres complètes - Tome IV(1927-1933). Paris : Payot ; 1982, pp. 125-135.

- Gutton Ph. (1991).Le pubertaire. Paris : Editions PUF - Le fil rouge, 322 pp.

- Gutton Ph. (1996).Adolescens. Paris : Editions PUF - Le fil rouge, 278 pp.

- Gutton Ph. (2000).Psychothérapie et adolescence. Paris : Editions PUF - Le fil rouge, $287 \mathrm{pp}$.

- Gutton Ph. (2008).Le génie adolescent. Paris :Editions Odile Jacob.

- Gutton Ph. (2008). Le pubertaire savant. In :Monographie de la revue Adolescence $\mathrm{n}^{\circ} 25$, pp. 347-358.

- Gutton Ph. (2013).Balthus et les jeunes filles - ou le dévoilement du féminin. Paris : Editions EDK médicales et scientifiques, 203 pp.

- Laplanche J. (1987).Nouveaux fondements pour la psychanalyse. Paris :Editions Quadrige.

- Rewald S. (1998). Balthus'sThérèses. In :Metropolitan Museum Journal, vol. 33. The University of Chicago Press, pp. 305-314.

- Rilke R. M. (1924). Dauer der Kindheit.In : Rainer Maria Rilke Werke - Band 2 : Gedichte 1910 bis 1926. (Briefwechsel mit Erika Mitterer in Die Gedichte 1922 bis 1926). Frankfurt am Main : Insel Verlag ; 1996, 971 pp.

- Rheims N. (2004).Le Rêve de Balthus. Paris : Editions Gallimard, 125 pp. 
$\boldsymbol{R} \boldsymbol{E} \boldsymbol{S U} \boldsymbol{M} \boldsymbol{E}$ (500 caractères, espaces compris)

Que viennent révéler les toiles de Balthus sur l'adolescence? A partir du dernier livre de Philippe Gutton intitulé Balthus et les jeunes filles, l'œuvre du peintre sera confrontée à la question de la métamorphose pubertaire féminine. Car tout comme le psychanalyste, l'artiste semble appréhender l'advenue du féminin pubertaire dans un contexte de féminité infantile phallique. Par ailleurs, nous nous placerons également du côté des jeunes modèles de Balthus en nous demandant à quoi elles rêvent.

\section{Mots clés}

\section{Pubertaire - féminin - Balthus}

